\title{
Metadata Challenges for Situational Properties of Learning Objects
}

\author{
Baden Hughes and Roderick A. Farmer \\ Department of Computer Science and Software Engineering \\ The University of Melbourne \\ Parkville, VIC 3010, Australia \\ \{badenh,raf\}@csse.unimelb.edu.au
}

\begin{abstract}
The abstraction of free-standing metadata describing learning objects is typified by an analytical model which primarily focuses on the encoding of discrete properties pertaining to the learning object itself. This process often neglects the contextual properties derived from the situations in which the learning object can be used optimally. We report the derivation of situated properties for learning objects through the use of the CASE framework and explore how these properties are best encoded.
\end{abstract}

\section{Introduction}

The creation of free-standing metadata describing learning objects has been the subject of considerable research. Standards for encoding learning object metadata such as IEEE Learning Object Metadata (LOM) [7] and SCORM [1] have emerged, and repositories of learning objects described within these frameworks are now publically available. However, reducing learning object properties to elements in a controlled vocabulary or taxonomy have largely resulted in discrete properties derived from learning object instantiations. In earlier work [6], we proposed the need to focus on the more holistic, socially-informed, and non-deterministic aspects of learning object design. We argued that learning is fundamentally a situated activity and demonstrated that learning objects can imbue these 'situated' characteristics, behaving as instructional descriptors for assisting in the aggregation of learning objects into larger, goal-oriented (or learning outcome) assemblies [5]. We proposed that these situated learning objects properties could be derived via application of the CASE framework [3, 4]. This paper considers the complexity of encoding learning object properties derived from situated learning frameworks, such as CASE, within existing learning object metadata description frameworks.

\section{Deriving Descriptors from CASE}

The CASE framework provides an integrative approach to the design of learning objects as learning tools. CASE provides a means of capturing salient aspects of cognition and social interaction within a situated learning paradigm [6]. CASE incorporates an iterative and activity-centric design meta-model, consisting of four concerns: Cognition, Activity, Social Organisation and Environment. Each concern, or component, represents a level of investigation. Likely influential factors impacting learning activities are situated within and across corresponding components of the framework [6]. Concerns are not mutually exclusive and may be used summatively or productively (depending on the desired analysis) to produce an indication of learnerinterface complexity at each level of abstraction. Representative descriptors for each CASE concern are shown below. Initially, CASE metadata would be drawn from learner characteristics in Instructional Design [8].

Cognition: task-type, attention, attitude, goals, aptitude, intelligence

Activity: experience, engagement-type, leadership, learning style, motivation, demographics

Social Organization: status, roles, responsibilities, value systems, peer relationships

Environment: materials, colocation, geographical location, affordances, control, perceived context

\section{Learning Object Metadata Frameworks}

A variety of descriptive frameworks for learning objects are in widespread use, including IEEE LOM, Sharable Courseware Object Reference Model (SCORM), and Dublin Core [2]. Considerable effort has been invested in the development of these standards, and in their adoption by teaching and research communities. This paper considers how these well established standards can encode these new situationally-derived learning object properties. 
Learning object metadata standards tend to focus on the minimal set of attributes which are needed to allow learning objects to be managed, located, and evaluated. There is recognition that these standards will likely be insufficient in all cases. As such, nearly all of them incorporate a mechanism for local extension of the basic fields and entity types, including optionality for given fields. These learning object metadata frameworks have as a core value the idea that object manipulation should be supported in the abstract, i.e. without specific inclusion of the object itself in the first instance. Services such as searching, sequencing and aggregation all build on this functionality to leverage resources at the level of metadata descriptions.

\section{Encoding CASE properties in IEEE LOM}

We have selected a single framework, IEEE LOM, into which we wish to encode these situational properties. We now turn to the actual task of inclusion. There are two main issues to consider: the relevent IEEE LOM element under which to include the properties and the representational data type.

One possibility is to include situational properties as free text within description. This has the obvious benefit of being human readable and easy to support, but inadequate for automated processing for tasks such as discovery, classification and assembly. Another possibility is to include sitational properties as new values within structure. In this case, the values selected are not likely to be the default atomic but rather a qualified selection of networked, hierarchical or linear.

The education element offers several possibilities. The first is as an attribute under interactivity type, selecting active rather than expository and qualifiying it with a new set of values. Another education possibility is within interactivity level, which for situationallyderived learning object properties is most likely to result in a selection of medium or high; neither of which without qualification are necessarily indicative of the properties themselves. The final education item would be as description, as free text. Similar points can be made here as were made earlier for description.

The next question to examine involves whether or not situationally derived properties should be free standing new elements, attributes of existing or new elements, or values for new or existing attributes. Some aspects of situationallybased descriptors do lend themselves to interpretation simply as sets of values (particularly where there is a singleton choice required to express a particular property). Others are more complex and require more than a single level, ie addition of more than just values, including the addition of elements and/or attributes. Largely the choice between one or another layer of addition is motivated by the complexity of the properties which need to be encoded; values which are either mutually exclusive, or purely additive are best expressed as controlled vocabularies for values. More complex entitities may require the addition of one or more attributes and associated values.

\section{Conclusion and Future Work}

We have considered the problems of encoding situationally derived properties for learning objects in learning object metadata standards such as IEEE LOM and note that some of these dynamic properties can be accounted for within existing metadata standards. Others, are somewhat more problematic. Future work on how best to reduce learning object properties to attributes which can be expressed in formal metadata is warranted. An obstacle in achieving this goal is that situationally-derived learning object properties do not easily conform to the object models of existing metadata standards.

\section{References}

[1] Advanced Distributed Learning. Sharable Content Object Reference Model (SCORM), 2004. http://www.adlnet.org/scorm/index.cfm.

[2] Dublin Core Metadata Initiative. Dublin Core Metadata Element Set 1.1: Reference Descrption, 2006. http://dublincore.org/documents/dces/.

[3] R. A. Farmer. Situated task analysis in learner-centred call. In P. Zaphiris, editor, User-Centred Computer Aided Language Learning. Idea Group Publishing, Hershey, PA. In Press.

[4] R. A. Farmer and B. Hughes. Case: A framework for evaluating learner-computer interaction in computer-assisted language learning. In Proceedings of CHINZ 2005 6th International Conference of SIGCHI-NZ, pages 67-74. ACM Press, 2005.

[5] R. A. Farmer and B. Hughes. A classification-based framework for learning object assembly. In Proceedings of the IEEE International Conference on Advanced Learning Technology (ICALT'05), pages 72-74. IEEE Computer Society Press, 2005.

[6] R. A. Farmer and B. Hughes. A situated learning perspective on learning object design. In Proceedings of the IEEE International Conference on Advanced Learning Technology (ICALT'05), pages 4-6. IEEE Computer Society Press, 2005.

[7] IEEE. 1484.12.1-2002 IEEE Standard for Learning Object Metadata.

[8] J.E. Kemp and G.R. Morrison and S.M. Ross. Designing Effective Instruction. John Wiley and Sons, 2001. 


\section{University Library}

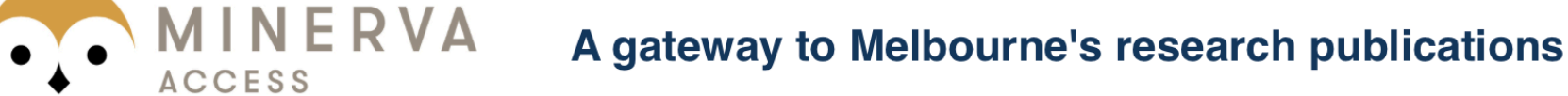

Minerva Access is the Institutional Repository of The University of Melbourne

Author/s:

HUGHES, B;FARMER, RA

Title:

Metadata Challenges for Situational Properties of Learning Objects

Date:

2006

Citation:

HUGHES, B. \& FARMER, R. A. (2006). Metadata Challenges for Situational Properties of Learning Objects. Proceedings of the 6th IEEE International Conference on Advanced Learning Technologies, pp.692-693. IEEE Computer Society.

Publication Status:

Published

Persistent Link:

http://hdl.handle.net/11343/34526 